\title{
The Fast Loss Electron Proton Instability
}

\author{
Michael Blaskiewicz* \\ AGS Department Brookhaven National Laboratory
}

\begin{abstract}
The fast loss electron proton instability is studied both experimentally and theoretically. It is shown that electron multi-pactoring is required for fast beam loss.
\end{abstract}

\section{Introduction}

Very fast, high frequency, transverse instabilities have been observed in the Los Alamos PSR $[1,2,3]$ and the AGS Booster[4]. The e-folding times ( $\sim 10$ turns) require a transverse resistance or order $5 \mathrm{M} \Omega / \mathrm{m}$ which is significantly larger than can be accounted for by the impedance budget. Additionally, the frequency of the instability depends strongly on beam parameters like beam intensity and betatron tune which implies a broad band impedance. Other observations, detailed below, also argue against an impedance driven phenomena.

An alternate instability mechanism is the interaction between the proton beam and electrostatically trapped electrons $[1,2,3,4,5,6,7,8,9,10]$. In the PSR and AGS Booster this e-p instability can result in losing more than half the beam in a few tens of microseconds. A less violent form of the ep instability was used to explain emittance growth in the ISR[10] and other machines have reported possible ep candidates. However, the PSR and AGS Booster are unique with the instability causing large losses in a short time.

\section{Data from the AGS Booster}

Instability studies in the AGS Booster were performed in 1998 and 1999. Data were taken with bunched beam using the normal magnet cycle and with coasting

"Work supported by the United States Department of Energy

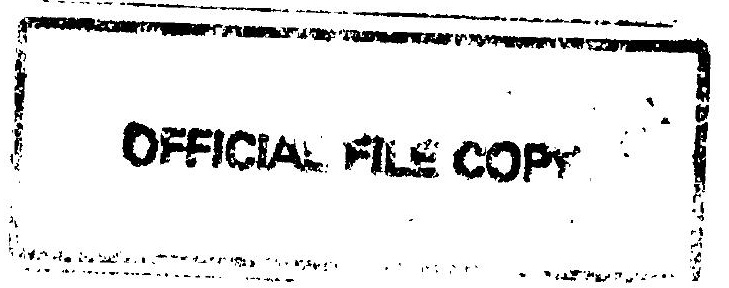


Table 1: machine parameters during study

\begin{tabular}{|c|c|}
\hline parameter & value \\
circumference & $2 \pi R=202 \mathrm{~m}$ \\
kinetic energy & $200 \mathrm{MeV}$ \\
frequency spread & $\pm 730 \mathrm{~Hz}$ \\
nominal betatron tunes & $Q_{x}=4.8, Q_{y}=4.95$ \\
beam pipe radius & $b=5 \mathrm{~cm}$ \\
injected beam radius & $\approx 3 \mathrm{~cm}$ \\
nominal chromaticity & $Q_{x}^{\prime}=-3, Q_{y}^{\prime}=-1$ \\
sextupoles off & $Q_{x}^{\prime}=-7.5, Q_{y}^{\prime}=-2.6$ \\
rf voltage & $0 \mathrm{~V}$ \\
linac RF frequency & $200 \mathrm{MHz}$ \\
injected pulse length & 200 to $450 \mu \mathrm{s}$ \\
revolution period & $1207 \mathrm{~ns}$ \\
\hline
\end{tabular}

beam and a magnetic field fixed at the injection value. The coasting beam data will be discussed.

Table 1 summarizes the machine parameters. The data consisted of current transformer traces which measured the total beam current, and wall current monitor traces which measure the $\mathrm{AC}$ longitudinal component. Also, horizontal and vertical split can capacitive beam position monitors (BPMs) were used to measure high frequency signals. The data from the BPMs were sampled at $1 \mathrm{GHz}$. The relative path lengths were measured to within $1 \mathrm{~ns}$ and sum and difference signals were obtained. Various processing techniques were applied to the BPM signals. Evidence of a violent instability with nominal values of tune and chromaticity and with intensity just above the threshold value is shown in Figure 1. The beam current shows a rapid loss beginning after the rise of the narrow band signal. The narrow band signal was obtained by applying a numerical filter to the vertical difference BPM signal. Recursive equations were used to reduce memory requirements.

$$
\begin{aligned}
F_{n+1} & =\left(\cos (\tilde{\omega} \tau) F_{n}-\sin (\tilde{\omega} \tau) G_{n}\right) e^{-\alpha \tau}+S_{n} \\
G_{n+1} & =\left(\sin (\tilde{\omega} \tau) F_{n}+\cos (\tilde{\omega} \tau) G_{n}\right) e^{-\alpha \tau} \\
P_{n+1} & =e^{-\tau / \tau_{0}} P_{n}+G_{n}^{2}
\end{aligned}
$$

In equations (1), (2), and (3) $S_{n}$ are the input data, $\tau=1 \mathrm{~ns}$ is the sampling interval, $\omega_{r}=\sqrt{\tilde{\omega}^{2}+\alpha^{2}}$ is the center frequency, $\alpha$ is the half width at half power bandwidth, and $\tau_{0}$ is an additional smoothing time on the output signal $P_{n}$.

The center frequency and bandwidth were obtained by examining Figure 2, which shows the evolution of the spectral amplitude of the vertical difference signal. 


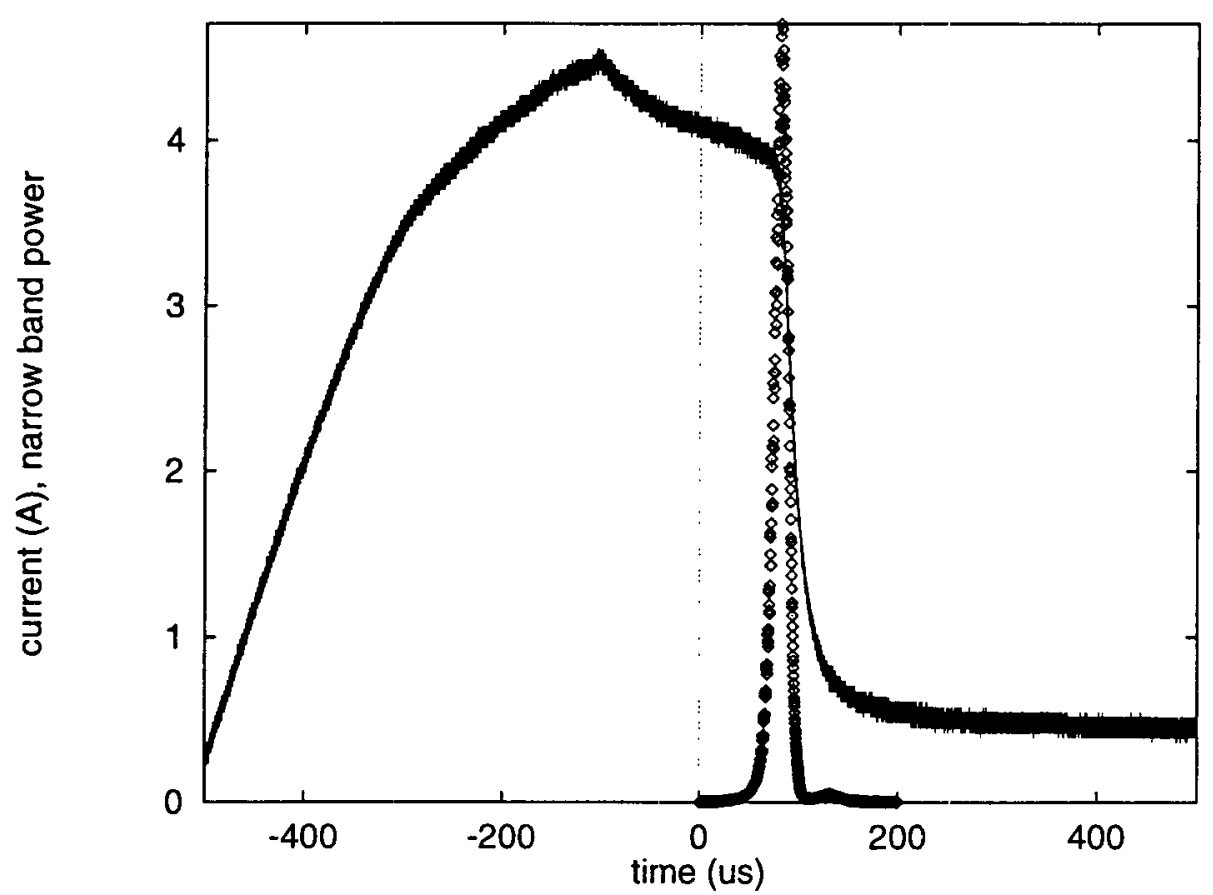

Figure 1: Beam current in amps and transverse power density measured using a capacitive BPM with bandpass between 75.6 and $76 \mathrm{MHz}$ and smoothed over 1 turn.

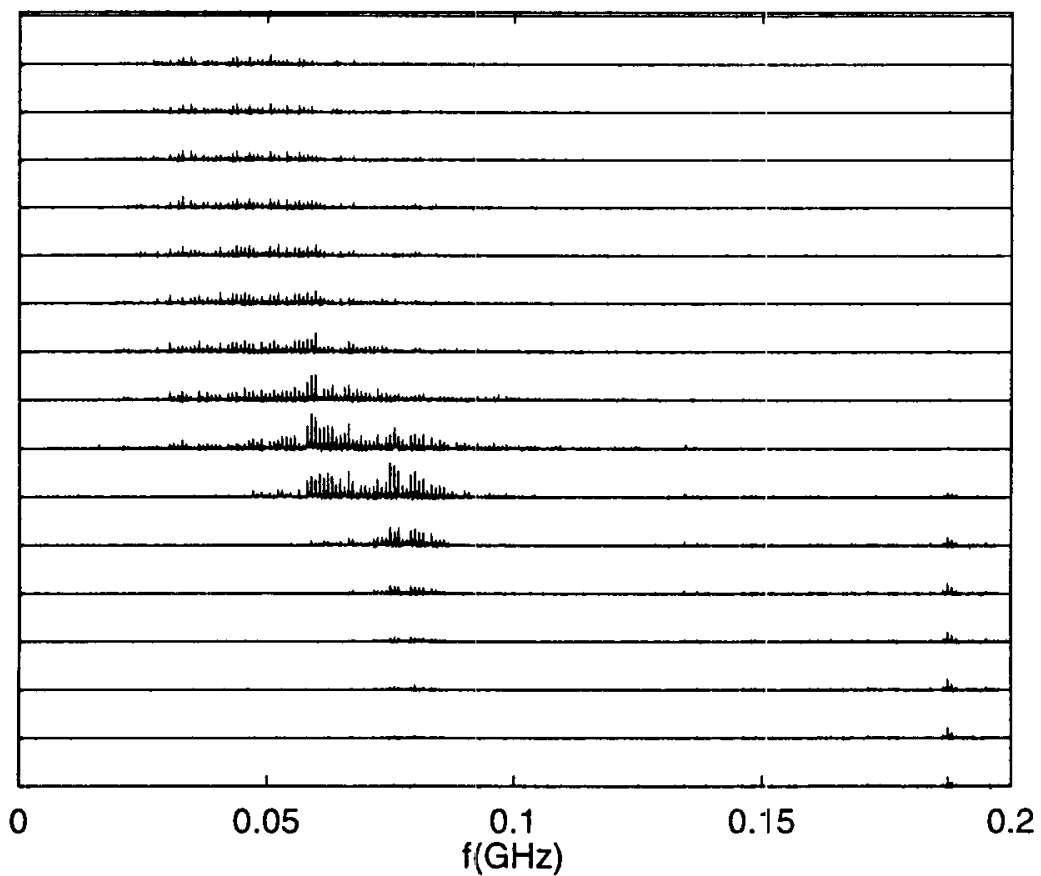

Figure 2: Spectral amplitude of vertical difference signal. The individual FFTs used ten turns of data ( $12 \mu$ s between traces). 
During the rise time of the instability the narrow band data are reasonably well fitted by an exponential with a $5.7 \mu \mathrm{s}$ e-folding time. Using the formula for transverse growth rates of a cold coasting beam,

$$
\operatorname{Im}(\Omega)=\frac{q c I_{\text {peak }} R e\left(Z_{\perp}\right)}{4 \pi E_{0} Q_{\beta}},
$$

an e-folding time of $2 \times 5.7=11.4 \mu \mathrm{s}$ implies $\operatorname{Re}\left(Z_{\perp}\right)=5.4 \mathrm{M} \Omega / \mathrm{m}$. Note that including frequency spread will increase the necessary impedance. Also, since many unstable lines are apparent in Figure 2 any driving impedance must be broad band as well. For comparison purposes the coherent transverse space charge impedance is

$$
-i \frac{R Z_{0}}{\beta^{2} \gamma^{2} b^{2}}=-i 8.4 \mathrm{M} \Omega / \mathrm{m} .
$$

With $\beta \gamma=0.69$ one expects transverse space charge to greatly dominate any other broad band impedance.

For other machine conditions the required impedance is larger still. Figure 3. shows the beam current and two narrow band signals. The vertical difference signal centered at $73 \mathrm{MHz}$ rises first, followed by the vertical sum signal. During the rise the vertical difference grows faster than exponentially. The instantaneous growth rate of the vertical signal, defined via $d \log P / d t$, peaks at $350 / \mathrm{ms}$. Using equation (4) a transverse resistance of $8.8 \mathrm{M} \Omega / \mathrm{m}$ is required.

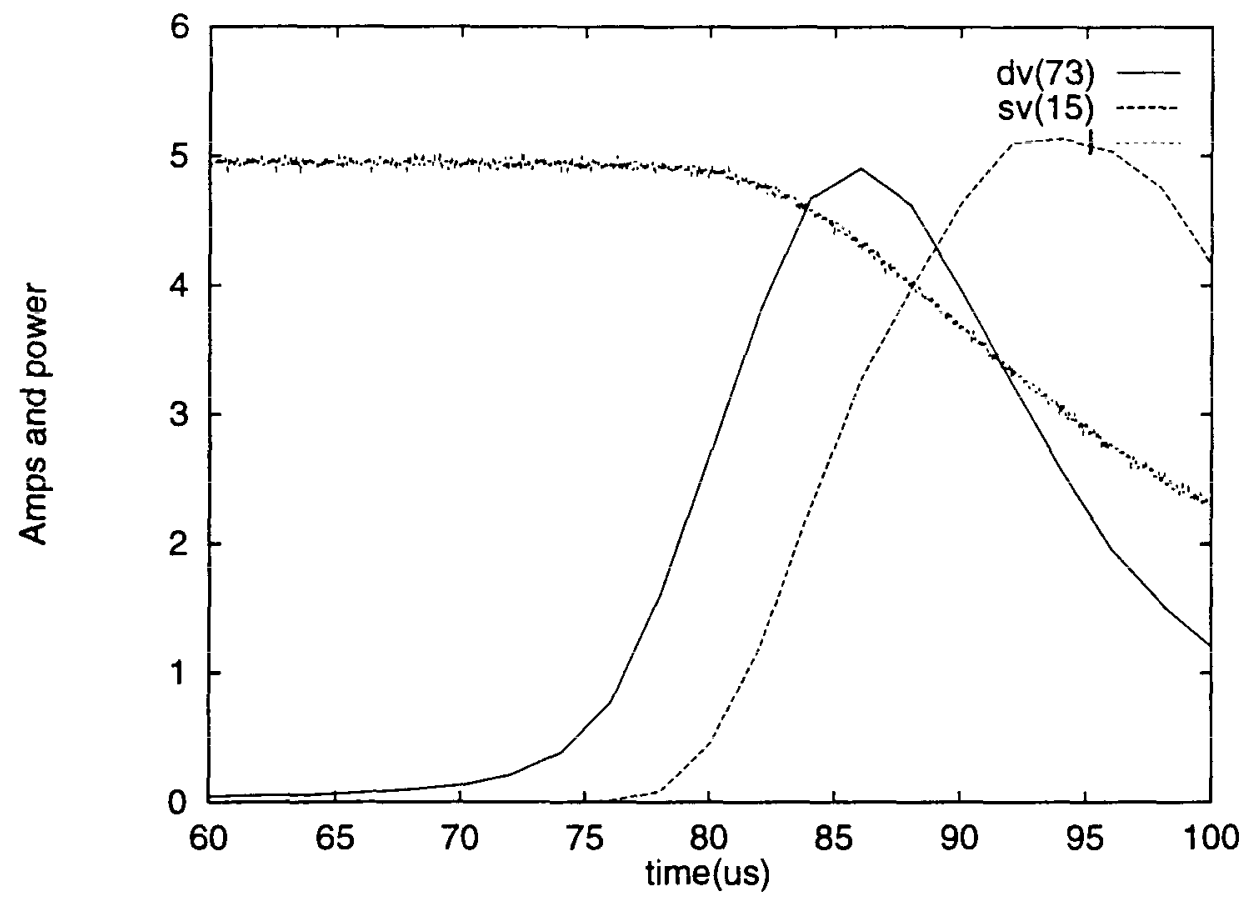

Figure 3: Beam current in amps, $P$ using vertical difference BPM centered at $73 \mathrm{MHz}$, and $P$ using vertical sum BPM centered at $15 \mathrm{MHz}$. Both are smoothed 
with $\tau_{0}=1.2 \mu \mathrm{s}$.

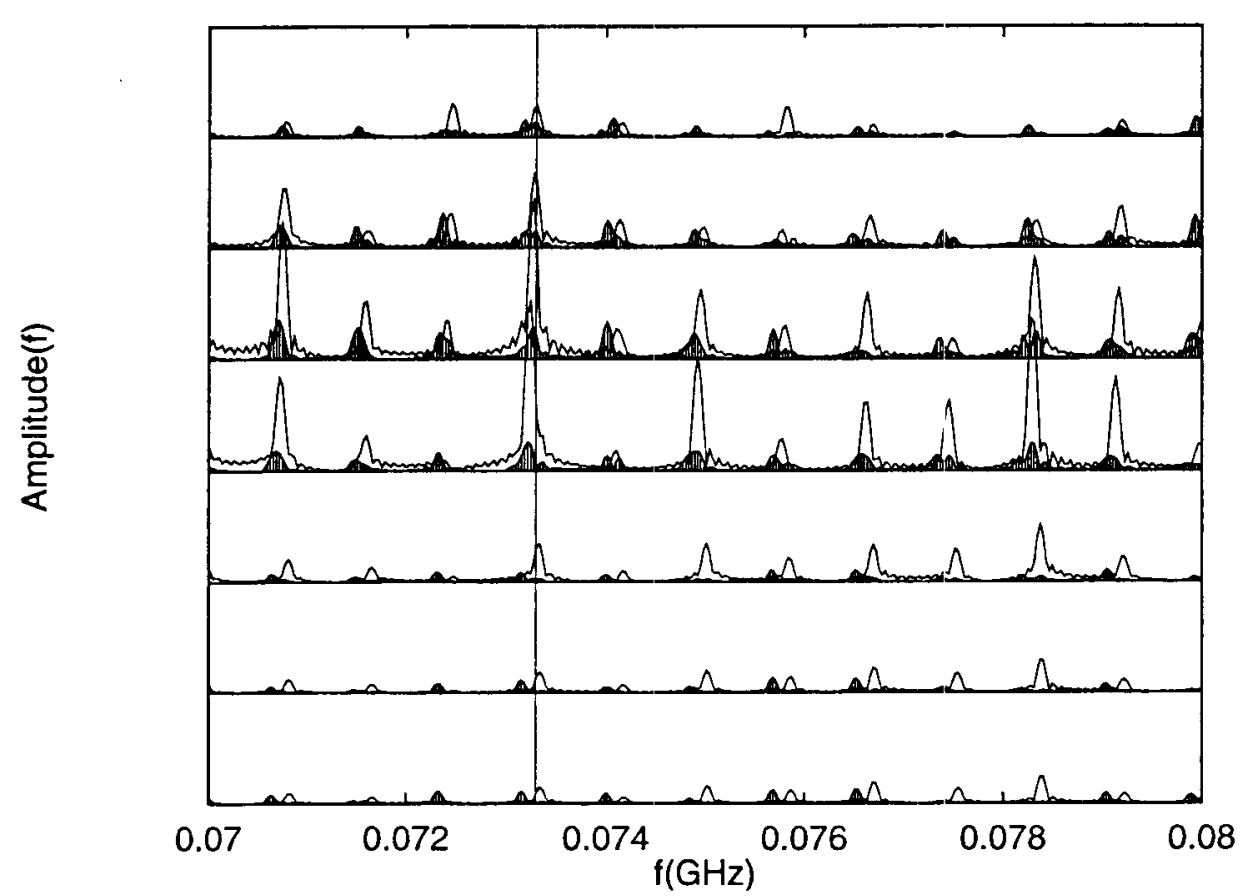

Figure 4: Spectral amplitude of vertical sum (cross hat:ched) and difference (smooth line) signals. The individual FFTs used ten turns of data $(12 \mu$ s between traces). The vertical line is at $73.3 \mathrm{MHz}$. The nearest vertical peak shifts down by $0.11 f_{\text {rev }}=$ $90 \mathrm{kHz}$ during the instability.

The setpoint tunes were $Q_{x}=4.75, Q_{y}=4.95$ and the sextupoles had zero current. The dependence of the instability threshold on tune was studied and it was found that these conditions had the largest threshold current. In particular, a smaller vertical tune did not increase the threshold. Figure 4 shows the evolution of the vertical sum and difference spectral amplitudes. There are several unstable lines implying that any driving impedance is broad band. From the figure it is also clear that the frequency of the peaks in the vertical difference spectrum shift down as the instability progresses. The shift is by $90 \mathrm{kHz}$ corresponding to a betatron tune shift of 0.1 which is very large given that the sextupoles were off. Additionally, it is difficult to imagine how such a strong impedance could be benign during normal operations. In the next section the coasting beam theory of the e-p instability is outlined and applied to these data.

\section{Coasting Beam Model}

The coasting beam theory in the linear limit with no electron multipactor has been carefully explored $[5,6,7,8]$. The model involves a coasting beam which traps 
particles of opposite sign in its electrostatic potential well. Some early work focused on electron beams trapping protons but the equations have good symmetry and are easily modified to the relevant case. There are several variables.

Let $\theta$ denote machine azimuth, $x$ be the horizontal coordinate, and $y$ the vertical. Assume the proton and electron beams have a uniform charge density and are round with radius $a$. Only transverse dipole motion is considered, since these modes are the most dangerous $[7,8]$. Let the proton beam have current $I$ and Lorentz factor $\gamma$. The line charge density of the protons is then $\lambda_{p}=I / v$. Take the fractional neutralization to be $f$, so that the line charge density of the electrons is $-f \lambda_{p}$. Assume the instability is vertical and take $\bar{y}_{p}(\theta, t)$ and $\bar{y}_{e}(\theta, t)$ to be the centroids of the proton and electron beams, respectively. Assume a round, perfectly conducting vacuum chamber of radius $b$. The $y$ component of the electric field due to the protons is

$$
E_{p}(x, y)=\frac{\lambda_{p}}{2 \pi \epsilon_{0}}\left(\frac{\left(y-\bar{y}_{p}\right)}{\max \left(\left(y-\bar{y}_{p}\right)^{2}+x^{2}, a^{2}\right)}-\frac{\left(y-b^{2} / \bar{y}_{p}\right)}{\left(y-b^{2} / \bar{y}_{p}\right)^{2}+x^{2}}\right) .
$$

The proton beam also creates a magnetic field $B_{x}=-\beta E_{p}(x, y) / c$. The electrons create a $y$ component of electric field,

$$
E_{e}(x, y)=\frac{-f \lambda_{p}}{2 \pi \epsilon_{0}}\left(\frac{\left(y-\bar{y}_{e}\right)}{\max \left(\left(y-\bar{y}_{e}\right)^{2}+x^{2}, a^{2}\right)}-\frac{\left(y-b^{2} / \bar{y}_{e}\right)}{\left(y-b^{2} / \bar{y}_{e}\right)^{2}+x^{2}}\right) .
$$

The electrons are non-relativistic and create negligible magnetic field. The vertical force on a proton is given by $F_{p}=e\left[E_{p}(x, y) / \gamma^{2}+E_{e}(x, y)\right]$ while the force on an electron is $F_{e}=-e\left[E_{p}(x, y)+E_{e}(x, y)\right]$

Analytically solving the Vlasov equation with the non-linear force terms in equations (5) and (6) is intractable. Linearizing the equations one finds $E_{p}(x, y)=$ $\left(\lambda_{p} / 2 \pi a^{2} \epsilon_{0}\right)\left[y-\bar{y}_{p}\left(1-a^{2} / b^{2}\right)\right]$ and $E_{e}(x, y)=\left(-f \lambda_{p} / 2 \pi a^{2} \epsilon_{0}\right)\left[y-\bar{y}_{e}\left(1-a^{2} / b^{2}\right)\right]$. For a non-round pipe there are additional geometrical factors associated with the quadrupole fields generated by a centered beam[7] but these are small and will be ignored.

Before considering the the general case assume that there is no frequency spread. The moment equations close [5] so only the average values of the coherent fields are relevant. The average force on a proton is given by

$$
\bar{F}_{p}(\theta, t)=\frac{e \lambda_{p}}{2 \pi a^{2} \epsilon_{0}}\left(\frac{a^{2}}{b^{2}} \frac{\bar{y}_{p}(\theta, t)}{\gamma^{2}}-f\left[\bar{y}_{p}(\theta, t)-\bar{y}_{e}(\theta, t)\right]-f \frac{a^{2}}{b^{2}} \bar{y}_{e}(\theta, t)\right) .
$$

The average force on an electron is

$$
\bar{F}_{e}(\theta, t)=-\frac{e \lambda_{p}}{2 \pi a^{2} \epsilon_{0}}\left(\frac{a^{2}}{b^{2}} \bar{y}_{p}(\theta, t)-\left[\bar{y}_{p}(\theta, t)-\bar{y}_{e}(\theta, t)\right]-f \frac{a^{2}}{b^{2}} \bar{y}_{e}(\theta, t)\right) .
$$


Seting $\bar{y}=Y(\theta, t)$ and applying the force laws give:

$$
\begin{aligned}
& \ddot{Y}_{p}=\left(\frac{\partial}{\partial t}+\omega_{0} \frac{\partial}{\partial \theta}\right)^{2} Y_{p}=-\omega_{\beta}^{2} Y_{p}+\bar{F}_{p}(\theta, t) / \gamma m_{p} \\
& \ddot{Y}_{e}=\left(\frac{\partial}{\partial t}\right)^{2} Y_{e}=\bar{F}_{e}(\theta, t) / m_{e} .
\end{aligned}
$$

Setting $Y_{e} \equiv 0$ in (7) and $Y_{p} \equiv 0$ in (8) yields electron and proton frequencies of

$$
\omega_{e}^{2}=\frac{e \lambda_{p}}{2 \pi a^{2} \epsilon_{0} m_{e}}\left(1-f \frac{a^{2}}{b^{2}}\right) .
$$

and

$$
\omega_{p}^{2}=\omega_{\beta}^{2}-\frac{e \lambda_{p}}{2 \pi a^{2} \epsilon_{0} \gamma m_{p}}\left(\frac{a^{2}}{\gamma^{2} b^{2}}-f\right) .
$$

Define the coupling frequencies

$$
\Omega_{e}^{2}=\frac{e \lambda_{p}}{2 \pi a^{2} \epsilon_{0} m_{e}}\left(1-\frac{a^{2}}{b^{2}}\right)
$$

and

$$
\Omega_{p}^{2}=\frac{f m_{e}}{\gamma m_{p}} \Omega_{e}^{2}
$$

With these definitions $\ddot{Y}_{p}=-\omega_{p}^{2} Y_{p}+\Omega_{p}^{2} Y_{e}$ and $\ddot{Y}_{e}=-\omega_{e}^{2} Y_{e}+\Omega_{e}^{2} Y_{p}$. The equations are linear with constant coefficients. Barring double roots, any unstable solution is a linear combination of [7]

$$
Y_{p}=\hat{Y}_{p} \exp \left(i n\left(\theta-\omega_{0} t\right)+i\left(\omega_{p}+\delta \omega_{p}\right) t\right)
$$

and

$$
Y_{e}=\hat{Y}_{e} \exp \left(i n \theta-i\left(\omega_{e}+\delta \omega_{e}\right) t\right) .
$$

Of course the frequencies are the same with $n \omega_{0}-\left(\omega_{p}+\delta \omega_{p}\right)=\omega_{e}+\delta \omega_{e}$.

Assume $\delta \omega_{p} \ll \omega_{p}$ and $\delta \omega_{e} \ll \omega_{e}$ then

$$
-2 \omega_{p} \delta \omega_{p} Y_{p}=\Omega_{p}^{2} Y_{e}, \quad-2 \omega_{e} \delta \omega_{e} Y_{e}=\Omega_{e}^{2} Y_{p}
$$

Solving for $\delta \omega_{p}$ yields

$$
\delta \omega_{p}=\frac{\Delta \omega}{2} \pm \sqrt{\left(\frac{\Delta \omega}{2}\right)^{2}-\frac{\Omega_{\psi}^{2}}{4 \omega_{p} \omega_{e}^{2}}}
$$


where $\Delta \omega=n \omega_{0}-\omega_{p}-\omega_{e}$. For unstable conditions $\left|\delta \omega_{e}\right|=\left|\delta \omega_{p}\right|$ and the ratio of proton to electron amplitudes is given by

$$
\left|\frac{\hat{Y}_{p}}{\hat{Y}_{e}}\right|=\sqrt{\frac{\Omega_{p}^{2} \omega_{e}}{\Omega_{e}^{2} \omega_{p}}}=\sqrt{\frac{f m_{e} \omega_{e}}{\gamma m_{p} \omega_{p}}} .
$$

Since $|\Delta \omega| \leq \omega_{0} / 2$ for some $n$ the beam will be unstable if the second term under the square root in the equation (14) is greater than $\omega_{0}^{2} / 16$. Substituting this condition in (15) gives $\hat{Y}_{p} \sim \omega_{0} \hat{Y}_{e} / \omega_{e} \ll \hat{Y}_{e}$. Hence, the electrons will reach the beam pipe radius before the proton beam gains a significant amplitude. If the electrons are lost at the walls the instability will stop and a slight increase in proton emittance will be observed, as in the ISR[10]. The instability seen in the AGS Booster and PSR is much more violent so there must be some mechanism to replace electrons which hit the walls.

An electron striking the beam pipe leads to secondary emission which can cause an electron cascade and beam loss. A key parameter is the kinetic energy the electron has when it strikes the wall. This is easily estimated by assuming an electron grazes the wall on one oscillation and hits it on the next. If only one side of the vacuum chamber is involved the electron velocity on impact is given by

$$
v_{e}=b \sqrt{4 \pi \omega_{e} \omega_{I}}\left(1+O\left(\sqrt{\omega_{I} / \omega_{e}}\right)\right)
$$

where $\omega_{I}=\operatorname{Im}\left(\delta \omega_{e}\right)$. Assuming $\Delta \omega=0$, the electron kinetic energy when striking the wall is

$$
K E_{e}=2 \pi m_{e} b^{2} \omega_{e} \omega_{I} \approx \pi m_{e}\left(\omega_{e} b\right)^{2} \sqrt{\frac{f m_{e}}{\gamma m_{p}} \frac{\omega_{e}}{\omega_{p}}},
$$

where the second equality neglects image terms. The largest growth rate seen in the AGS Booster gives $\omega_{I}=175 / \mathrm{ms}$. With $\omega_{e} / 2 \pi=80 \mathrm{MHz}$ and $b=5 \mathrm{~cm}$ the first expression for the electron kinetic energy gives $K E_{e}=7.8 \mathrm{eV}$. This is a small kinetic energy and does not lead to a secondary emission yield greater than 1 . However, the validity of the coasting beam dispersion relations is an open issue. Suppose for the moment that the electron cloud uniformly fills the pipe. The proton beam will suffer a frequency shift of

$$
\delta \omega_{p}=\frac{f e I Z_{0}}{4 \pi \beta \gamma m_{p} c^{2} \omega_{\beta}} \frac{c^{2}}{b^{2}} .
$$

Setting this to the observed shift of $2 \pi \times 90 \mathrm{kHz}$ gives $f \approx 4$. Setting $f=4$ in the second expression of equation (17) results in $K E_{e} \approx 4 \mathrm{keV}$. Clearly, the theory is of limited value. Including frequency spreads will not help beyond the Landau damping threshold, so understanding multi-pactoring requires a non-linear theory. 
A theory with multi-pactoring may be obtained by letting $i \delta \omega_{p} \rightarrow d / d t$ in equations (13).

$$
\begin{aligned}
& \dot{X}_{p}=-i \frac{\Omega_{p}^{2}}{2 \omega_{p}} X_{e}, \\
& \dot{X}_{e}=i \Delta \omega X_{e}+i \frac{\Omega_{e}^{2}}{2 \omega_{e}} X_{p},
\end{aligned}
$$

where $X_{p}$ and $X_{e}$ are the slowly varying parts of $Y_{p}$ and $Y_{e}$, respectively. These equations can be obtained by setting $Y=X(t) \exp \left(i n\left[\theta-\omega_{0} t\right]+i \omega_{p} t\right)$ in the coupled oscillator equations and neglecting the $\ddot{X}$ terms. When multipactoring is included, equations (19) and (20) must be supplemented by the conditions that $\left|X_{\varepsilon}\right| \leq b$ and the frequencies become time dependent due to variation in the fractional neutralization $f$. In particular, $\Delta \omega$ will change with $f$ due to the presence of the image term in (9). Suppose equations (19) and (20) are evolved until $\left|X_{e}\right|=b-\epsilon$, where $\epsilon$ is a small numerical factor. Let $X_{e} \rightarrow X_{e} b /\left|X_{e}\right|$ to limit the electron amplitude. To get the electron velocity when striking the wall use equation (20) to obtain $\omega_{I}=\operatorname{Real}\left(\dot{X}_{e} / X_{e}\right)$. If $\omega_{I}>0$, which is not always the case for $\left|X_{e}\right|>b-\epsilon$, use equation (16) to get the electron kinetic energy, $E_{k}$. The change in $f$ with respect to time depends on the secondary emission yield, $S E Y\left(E_{k}\right)$ via,

$$
\dot{f}=f \frac{\omega_{e}}{2 \pi}\left(S E Y\left(E_{k}\right)-1\right)-D(f),
$$

where the factor $\omega_{e} / 2 \pi$ implies that electrons hit the wall on every oscillation after multipactoring occurs, and the term $D(f)$ is present since when $f \gtrsim 1$ the mutual electrostatic repulsion of the electrons expels them from the vacuum chamber[11]. In the numerical work I simply set $f \rightarrow 1$ when the updated value was greater than 1. The $S E Y$ depends on the chamber material and on the incidence angle of the electrons [11]. Bellows and sharp transitions in pipe radius yield enhanced values of $S E Y$. Additionally, if the electrons hit both vacuum chamber walls equation (16) overestimates $E_{k}$ by a factor of 2 and $\omega_{e} \rightarrow 2 \omega_{e}$ in equation (21). Therefore, any specific numerical results are of limited value.

Some general trends in the simulations of the AGS Booster have been observed.

- The system is usually unstable if $S E Y>1$ the first time it is called. In some cases, a first value of $S E Y=0.8$ results in unstable behavior after a few microseconds.

- The shift in $\Delta \omega$ with $f$ does not cause the system to stabilize after multipactoring begins.

- The image terms can reduce $E_{k}$ by a factor of 2 below the value obtained ignoring images. 
- During instability, while $f$ is increasing, the growth in $\left|X_{p}\right|$ is roughly linear with time.

- For peak $S E Y \sim 2$ and $f \lesssim 0.1$ initially, the characteristic time from the onset of multipactoring to $\left|X_{p}\right|=b$ is of order ten microseconds. (For numerical simulations I set $X_{p}(0)=0$ and $X_{e}(0) \sim b / 10$.)

- Reducing the peak value of $S E Y$ increased the threshold value of $f$.

- Increasing the energy at which $S E Y$ peaks increases the threshold value of $f$.

\section{Conclusions}

Instabilities observed in coasting beam data exhibit transverse growth rates $~$ $100 / \mathrm{ms}$. Assuming normal instabilities, broad band resistive components of order $5 \rightarrow 10 \mathrm{M} \Omega / \mathrm{m}$ are implied. When viewed in terms of the linear coasting beam electron proton instability the observed growth rates are too small. Adding multipactoring and limiting the electron amplitude to remain within the beam pipe yield a theory in reasonable agreement with the data.

This work has benefited from discussions with Thomas Roser and Y.Y. Lee.

\section{References}

[1] D. Neuffer et. al. NIM A321 p1 (1992).

[2] M. A. Plum et. al PAC97 p 1611.

[3] V. Danilov et.al PAC99 TUA 52.

[4] M. Blaskiewicz PAC 99 TUP 60.

[5] B.V. Chirikov, Sov. Atomic Energy, 19 p1149 (1965).

[6] E. Keil, B. Zotter, CERN/ISR-TH/71-58, (1971).

[7] L. J. Laslett, A. M. Sessler, D. Möhl, NIMA, 121, p517, 1974.

[8] R. C. Davidson, H. Qin, P. H. Stoltz, Phys Rev ST Accel Beams, 054401, 1999.

[9] A. Ruggiero, M. Blaskiewicz, PAC 97 p1581 (1998).

[10] H. G. Hereward, CERN 71-15 (1971).

[11] G.V. Stupakov, LHC Project Report 141 (1997). 